\title{
Local Immunization Based on Degree- Community-Bridge-Find in Heterogeneity Community Networks
}

\author{
Kai Gong \\ School of Computer Science and Engineering \\ University of Electronic Science and Technology of China \\ Chengdu 610054, China \\ gongkai1210@gmail.com
}

\begin{abstract}
Identifying and immunizing nodes in networks with heterogeneity community is very important to prevent epidemic spreading. The effectiveness of preventing the transmission of infectious disease can be greatly enhanced by choosing suitable nodes to vaccinate. Community structure can amplify the influence of a small number of bridge nodes which maintain the linking in different communities. However, in heterogeneity networks with community structure, identifying bridge nodes which overlap with different communities are not achievable with traditional global immunization algorithms. To consolidate the efficiency of immunization, it is essential to utilize the community topology and identify bridge nodes. Here, we develop an effective local algorithm, the Degree-CommunityBridge-Find, to identify and immunize bridge nodes. Results show that Degree-Community-Bridge-Find generally outperforms Community-Bridge-Find in heterogeneity community networks.
\end{abstract}

Keywords- community; immunization algorithm; epidemic dynamic model

\section{INTRODUCTION}

Since the spread of infectious diseases (e.g., SARS in 2002, Avian influenza in 2003, and H1N1 in 2009) caused more and more serious harm and economic loss in modern society $[1,2]$ how to prevent epidemic spreading in time has been attracting wide attentions from different research fields[3-6]. As all know, immunization and quarantine are two basic measures to prevent epidemic spreading. In the face of epidemic outbreaks, it is a very important problem to identify who will be immunized or quarantined.

In complex network, an individual with high centrality, which is called hub, has a significant impact on the dynamics of epidemics. In contrast to common belief, the best spreaders do not correspond to the most highly connected individuals. Instead, Kitsak[7] argued that nodes with high $\mathrm{k}$-shell are more efficient spreaders in some real networks. Thus it is clear that immunizing hubs can prevent epidemic spreading effectively. To propose efficient immunization strategy, it is necessary to identify hubs in complex networks. Up to now, these studies fall into two topics: global and local immunizations. For the global immunization, hubs may be identified by different centrality measures, such as degree centrality[8], eigenvector centrality[9], betweenness centrality[10].
However, this approach is unrealistic for large scale networks because it requires a complete knowledge of all nodes. That is one reason why local algorithm is more applicative in practice when used in large scale networks. For example, random walk algorithm is widely applied to scale-free networks[11], and acquaintance immunization is efficient for large networks of any broad-degree distribution[12]. Furthermore, epidemic outbreaks can be minimized by fragmenting the networks via a graph partition algorithm which requires less immunization ratio[13]

Community structures at mesoscale level are ubiquitous in a variety of real complex systems[14], such as Facebook[15], and Twitter[16]. Many recent studies have contributed to understanding how community structures affect the dynamics of epidemic[17]. Except for the hub, bridge node is another kind of important node in community networks[18]. However, the meaning of bridge node may differ in different kind of networks[19]. For example, the bridge nodes can be words with multiple meanings in world association networks. And, in protein networks, a protein with multiple roles can be bridge node. In this paper, the bridge node is defined as an individual connecting different communities in network. The bridge node provides ways between different communities for the diffusion of information and disease[20]. In particular, the bridge node has been proved to be more effective than the hub in the diffusion of information through community networks[21]. Therefore, if bridge nodes can be identified in community networks, it is able to provide an useful reference for preventing epidemic outbreaks. In Ref.[22], these algorithms used to identify bridge nodes can be divided into two types: deterministic and stochastic algorithms. The former requires the complete information about each node, like global immunization, which greatly limits its applications and also limits its processing speed. In order to overcome this short, stochastic algorithm has been proposed, which does not require the complete structural information. Salathe et al. [22] generalized that immunization interventions targeted at bridge nodes which linking communities are more effective than those hubs. In their paper, Community-Bridge-Finder ( $\mathrm{CBF})$, which is based on local information, has been developed by using random walk algorithm to identify the bridge nodes. 
In this study, we propose an simple immunization algorithm in heterogeneity networks with community structures. We then show how our algorithm, which we call it Degree-Community-Bridge-Find, can be applied to heterogeneity communities. It gives a better efficiency of controlling the outbreak of epidemics in networks which the community structure is obvious.

\section{SIMULATED NETWORK}

The heterogeneity simulated community networks of different modularity $\mathrm{Q}$ are generated by the algorithm given in Ref.[22]. There are $m$ independent scale-free communities. In each community, $\mathrm{n}$ nodes are randomly connected so that the mean degree is $k_{\text {in }}$. These communities are then connected randomly by $\mathrm{E}_{\text {out }}$ links. The simulated community network thus has a total of mn nodes and $\mathrm{mn}^{*} \mathrm{k}_{\mathrm{in}}+\mathrm{E}_{\mathrm{out}}$ undirected links. We used the same set of parameters in this paper, namely $m=10, n=500$, $\mathrm{k}_{\mathrm{in}}=6$, and $\mathrm{E}_{\mathrm{out}}=2000$. After generating the network, the modularity $\mathrm{Q}$ can be evaluated according to the definition given in Ref.[14]. The modularity $Q$ can be varied by rewiring some inter-community links into intra-community links, following the rewiring procedures given in preference rule:

$$
P(\mathrm{i})=\left[\frac{k_{i}}{\Sigma_{j=1, j \neq i} k_{i}}\right]^{\alpha}
$$

Therefore, a node that is a neighbor of many other nodes, i.e., a hub, will have higher chance to be chosen for connection. In the simulation, the parameters are taken to be $\alpha=1$.

\section{EPIDEMIC DYNAMIC}

We have implemented the classical susceptibleinfected-recovered(SIR) model[23], in this model individuals can only exist in three possible states: (S)usceptible, (I)nfected, (R)ecovered. The healthy individuals are susceptible(S) to infection, I denotes the individuals who have been infected and are capable to spread to others in the susceptible, and the individuals who are recovered(R)from the infection are not able to be infected again or to transmit to others. In the beginning, all nodes are susceptible.

An epidemic simulation apply those algorithms before the infection occurs. Identified nodes are chosen by different immunization algorithms until vaccination coverage of nodes is achieved, then set their states as recovered.

After that one susceptible node is randomly picked up and become infected. The epidemic simulation rules are: (1) Each susceptible node can be infected with probability 1$\mathrm{e}^{\left(-\beta^{*} i\right)}$ at each step, where $\beta=0.08$ is the transmission rate, parameter $i$ is the number of current infected neighbors. (2) In every step, infected nodes are assumed to recover with a constant probability $\mu=1$. (3)The process of epidemic simulation stops once there are no further infected nodes.
In order to implement the synchronous updating scheme states of all nodes in network are updated at the same time.

\section{DEGREE-COMMUNITY-BRIDGE-FIND}

In this section, we review existing method for immunizing in local communities and discuss the ways in which these approaches may fail, before describing our method. First, we briefly describe one local algorithms: Community-Bridge-Find, with which we compare our algorithm results. CBF which identify nodes that connect to different communities without complete information, does based on the notion that the first node that does not connect back to previously visited nodes is likely to be a bridge node between different communities, and represent a random walk on networks. We have implemented CBF following the algorithm in Ref.[22], where it has been shown that, without prior knowledge of the community structure, $\mathrm{CBF}$ is more efficient than other local immunization strategies that target at the different kinds of hubs.

Bridge nodes are important for transmission through heterogeneity communities. Like CBF, DCBF is an algorithm based on self-avoiding walks. The different is: two nodes are not randomly chosen among all the possible nodes that the walk could go in steps, but according to preference rule from high to low. i.e., two high degree neighbors are chosen from all the neighbors due to the self-avoiding restriction of the walk. In the case that there is only one neighbor to choose from, the only neighbor will be considered. In practice, two additional checks are implemented to shorten the computing time. Firstly, the number of nodes registered in a running path is kept at the length of ten, using the latest ten nodes visited. Secondly, the number of visits by any random walk for each node is recorded. When the number $k$ of visits equals a certain number $(k=2)$, the node is immunized.

\section{RESULTS}

To test the efficiency of immunization strategies on networks, we generate computationally network with communities by creating scale-free networks. Then we run standard susceptible-infected-resistant(SIR) epidemic model on these networks.

In the computationally generated networks, DCBF is more advantage than $\mathrm{CBF}$ algorithm in controlling outbreak at large areas of the parameter space, see Fig.1 The difference in the final epidemic ratios(\%) between DBHD(Fig.1a) and CBF(Fig.1b), are shown for simulated networks with different network modularity $\mathrm{Q}$ and immunization coverage $f(\%)$. The colors indicate the differences in percentages (see color codes). Results are obtained by averaging over times 2000 realizations for each pair of $\mathrm{Q}$ and $f$. The parameters associated with the SIR dynamics are $\beta=0.08$ and $\mu=1$. It is important to note that outbreaks will be restricted to local community when $\mathrm{Q}$ is high. Thus, CBF algorithm unfortunately is not an effective way to control the spreading of epidemics because immunizing bridge nodes is useless. However, DCBF may at least identify highly connected nodes in local community and will perform better than $\mathrm{CBF}$ under the extreme condition $(\mathrm{Q}>0.84)$. 

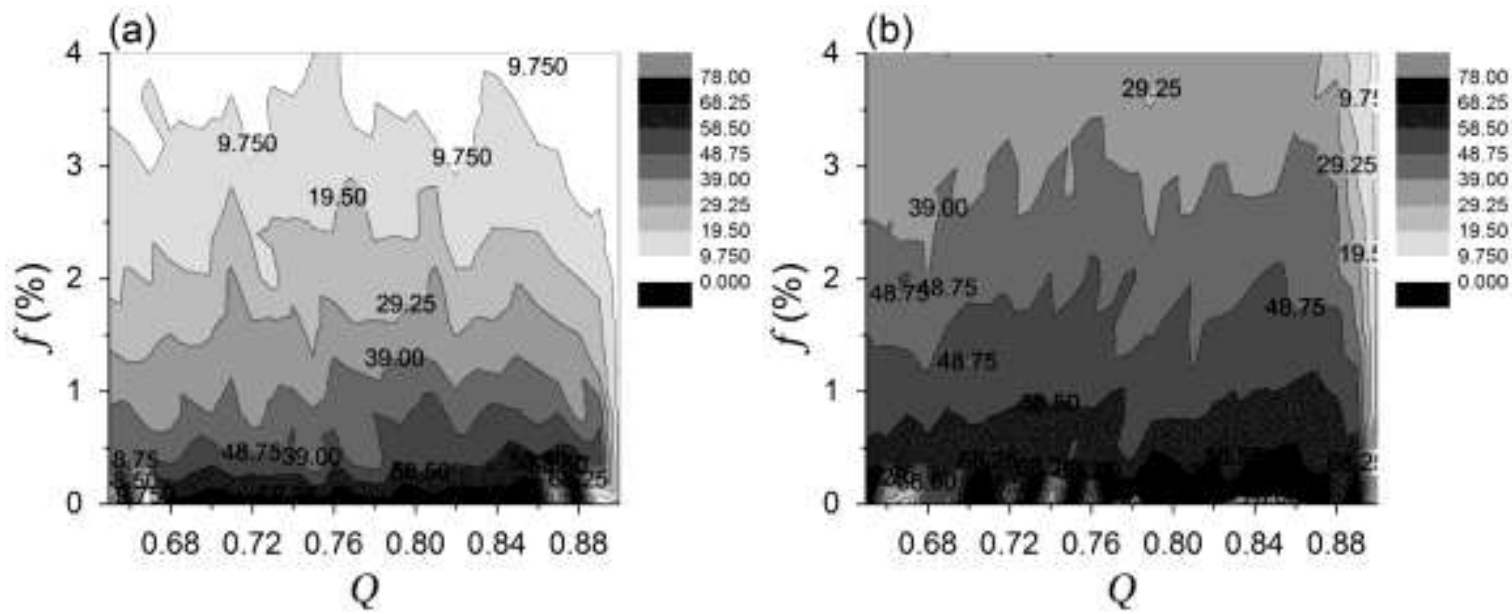

Figure 1. Comparison of efficacy of immunization algorithms in simulated networks. Color code denotes the difference in the average final size of disease outbreaks in networks that were immunized before the spreading using different algorithms. The left panels(a) show the DCBF, and the right panels(b) measuring the CBF. Average results were obtained by testing 2000 simulations, $\beta=0.08$ and $\mu=1$.

The difference in the peak (\%) between DBHD(Fig.2a) and $\mathrm{CBF}$ (Fig.bb), are also shown for simulated networks with different network modularity $\mathrm{Q}$ and immunization coverage $f(\%)$, like Fig.1. Results are obtained by averaging over times 2000 realizations for each pair of Q and $f$. The parameters associated with the SIR dynamics are $\beta=0.08$ and $\mu=1$. The results for the differences in peak prevalence are given in Fig. 2 and the features are essentially the same as those in Fig.1
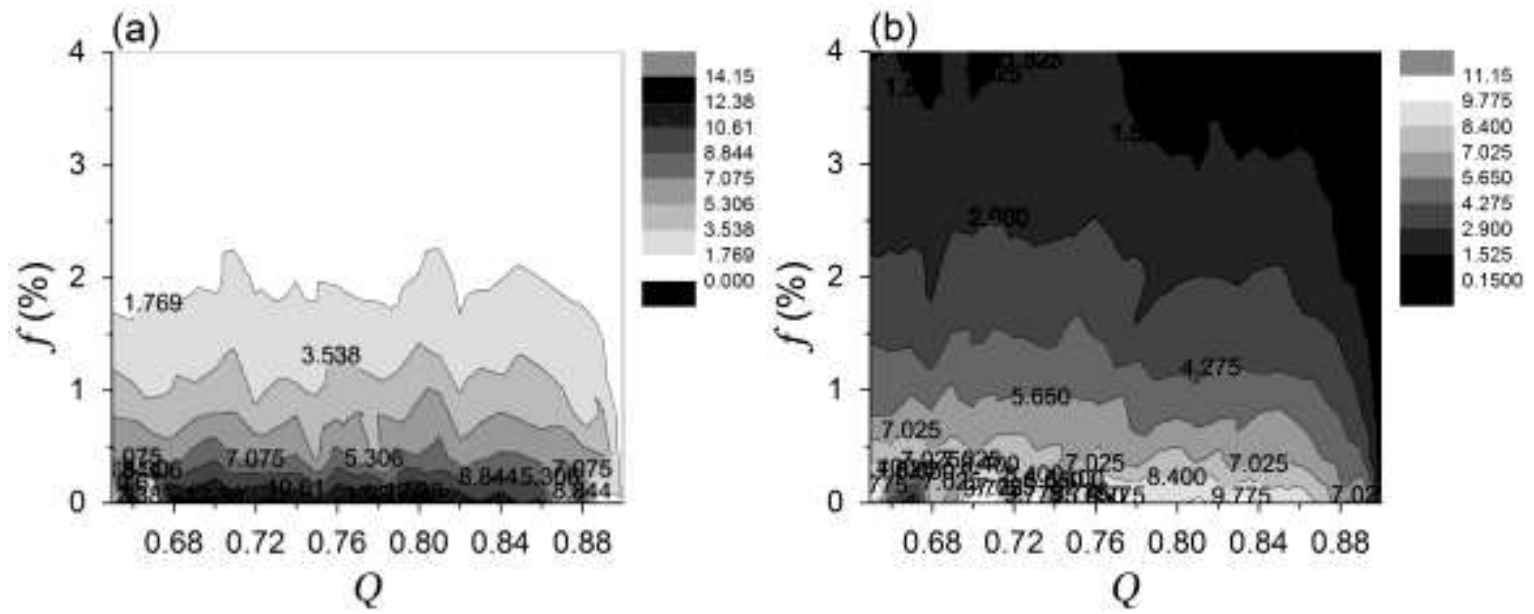

Figure 2. Comparison of peak prevalence of immunization algorithms in simulated networks. Color code denotes the difference in the average peak of disease outbreaks in networks that were immunized before the spreading using different algorithms. The left panels(a) show the DCBF, and the right panels(b) measuring the $\mathrm{CBF}$. Average results were obtained by testing 2000 simulations, $\beta=0.08$ and $\mu=1$

\section{DISCUSSION}

After going through the above details, we may conclusion that developing an algorithm based on network with community to identify bridge nodes is not an easy task. It is not merely resulting who is the best, but also to consider the feasibility of immunizing in networks. The feasibility is very important to community networks, which are fundamentally different structure from scalefree networks. Therefore, immunization algorithm in networks with community should be easy manipulations in real time.

We suggest that Degree-Community-Bridge-Find may provide a scientific methodological approach for making controls of the spreading of epidemic in network with community. Furthermore, this algorithm generally outperforms at several important features. Firstly, we show in this paper that DCBF identify bridge nodes which can cause extensive spreading of infection, and is of particular importance for networks with strong community structure. Secondly, aiming at the drawback of global algorithm, DCBF is local but simple immunization algorithm, one only needs information about the neighbors of a given node. All results make DCBF a good candidate for immunization algorithm in community networks.

Although DCBF did well performance for immunization as one local algorithm, this may need to further improvement to deal with tricky situations. For instance, the role of bridge nodes would be more prominent and intricate if node contains multiple "labels" that belongs to many groups, according to the definition of 
communities or other criteria. In cases where bridge nodes can be characterized by different categories, then multiple bridge nodes with multiple labels can be introduced. Such complex applications would require further investigations. On the other hand, DCBF can also be generalized to applications ranging from bridge nodes identification in social networks to community detection in graphs, and it could be lead to modifications of the algorithm for substantial improvements in performance.

Identifying bridge nodes in communities is still a problem. As accompanied by the spreading of the disease in human society, bridge identification may reinforce their efficiency for immunization. But, to be sure that the efficiency of such an immunization algorithm depends crucially on the structure of network. In addition, technological advance will provide more information to find bridge nodes that overlapped with different groups, at the same time will increase the complexity and scale up the network size that both make immunization task more challenging in the near future.

\section{ACKNOWLEDGMENT}

K. Gong acknowledges the support from the Program of Outstanding $\mathrm{PhD}$ Candidate in Academic Research by UESTC (Grant No. YBXSZC20131027).

\section{REFERENCES}

[1] J. H. Jones and M. S. Handcock, "Sexual contacts and epidemic thresholds," Nature, vol. 423, pp. 605-606, Jun 52003.

[2] B. Grenfell and O. Bjrnstad, "Sexually transmitted diseases Epidemic cycling and immunity," Nature, vol. 433, pp. 366-367, Jan 272005.

[3] R. Pastor-Satorras and A. Vespignani, "Epidemic spreading in scale-free networks," Physical Review Letters, vol. 86, pp. 32003203, Apr 2001.

[4] M. Barthelemy, A. Barrat, R. Pastor-Satorras, and A. Vespignani, "Velocity and hierarchical spread of epidemic outbreaks in scalefree networks," Physical Review Letters, vol. 92, Apr 302004.

[5] M. E. J. Newman, "A measure of betweenness centrality based on random walks," Social Networks, vol. 27, pp. 39-54, Jan 2005.

[6] O. Diekmann and J. A. P. Heesterbeek, Mathematical Epidemiology of Infectious Diseases: Model Building, Analysis and Interpretation: Wiley, 2000.

[7] M. Kitsak, L. K. Gallos, S. Havlin, F. Liljeros, L. Muchnik, H. E. Stanley, and H. A. Makse, "Identification of influential spreaders in complex networks," Nature Physics, vol. 6, pp. 888-893, Nov 2010 .
[8] R. Pastor-Satorras and A. Vespignani, "Immunization of complex networks," Physical Review E, vol. 65, Mar 2002.

[9] M. Boguna, R. Pastor-Satorras, and A. Vespignani, "Epidemic spreading in complex networks with degree correlations," Statistical Mechanics of Complex Networks, vol. 625, pp. 127-147, 2003.

[10] H. F. Zhang, K. Z. Li, X. C. Fu, and B. H. Wang, "An Efficient Control Strategy of Epidemic Spreading on Scale-Free Networks," Chinese Physics Letters, vol. 26, Jun 2009.

[11] K. Hu and Y. Tang, "Immunization for scale-free networks by random walker," Chinese Physics, vol. 15, pp. 2782-2787, Dec 2006.

[12] R. Cohen, S. Havlin, and D. ben-Avraham, "Efficient immunization strategies for computer networks and populations," Physical Review Letters, vol. 91, Dec 122003.

[13] Y. P. Chen, G. Paul, S. Havlin, F. Liljeros, and H. E. Stanley, "Finding a better immunization strategy," Physical Review Letters, vol. 101, Aug 12008.

[14] M. Girvan and M. E. J. Newman, "Community structure in social and biological networks," Proceedings of the National Academy of Sciences of the United States of America, vol. 99, pp. 7821-7826, Jun 112002.

[15] A. L. Traud, E. D. Kelsic, P. J. Mucha, and M. A. Porter, "Comparing Community Structure to Characteristics in Online Collegiate Social Networks," Siam Review, vol. 53, pp. 526-543, 2011.

[16] B. Goncalves, N. Perra, and A. Vespignani, "Modeling Users' Activity on Twitter Networks: Validation of Dunbar's Number," Plos One, vol. 6, Aug 32011.

[17] R. Guimera and L. A. N. Amaral, "Functional cartography of complex metabolic networks," Nature, vol. 433, pp. 895-900, Feb 242005.

[18] A. Clauset, M. E. J. Newman, and C. Moore, "Finding community structure in very large networks," Physical Review E, vol. 70, Dec 2004

[19] T. Nepusz, A. Petróczi, L. Négyessy, and F. Bazsó, "Fuzzy communities and the concept of bridgeness in complex networks," Physical Review E, vol. 77, p. 016107, 2008.

[20] E. Ferrara, P. De Meo, G. Fiumara, and A. Provetti, "The role of strong and weak ties in $\{$ Facebook $\}$ : a community structure perspective," ed, 2012. unpublished

[21] J. P. Onnela, J. Saramaki, J. Hyvonen, G. Szabo, D. Lazer, K. Kaski, J. Kertesz, and A. L. Barabasi, "Structure and tie strengths in mobile communication networks," Proceedings of the National Academy of Sciences of the United States of America, vol. 104, pp. 7332-7336, May 12007.

[22] M. Salathe and J. H. Jones, "Dynamics and Control of Diseases in Networks with Community Structure," Plos Computational Biology, vol. 6, Apr 2010

[23] M. J. Keeling and P. Rohani, Modeling infectious diseases in humans and animals. Princeton: Princeton University Press, 2008. 